\section{The Appropriate Testing Methods for OR Scrub?}

\section{To the Editor:}

I have some comments to add to the article "Serratia marcescens contamination of antiseptic soap containing triclosan: Implications for nosocomial infections" published in the September 1984 issue. $^{1}$

The subject of this article is $O R$ Scrub (Huntington Laboratories, Inc, Huntington, IN). The efficacy of OR Scrub as a surgical scrub and as a health care personnel handwash has been demonstrated by a number of in vivo studies such as the Peterson Glove Juice Test, the Price-Cade Basin Test, the Health Care Personnel Handwash Test, and specific clinical studies in the operating suite. Barry et al used in vitro modified preservative tests. ${ }^{1}$

First, "the recent investigation" was conducted in late 1982 and early 1983 and was first reported at the May 1983 APIC poster session. ${ }^{2}$ The authors found that 2 of 23 bottles of OR Scrub in use were contaminated with Serratia marcescens at 100 and $600 \mathrm{CFU} / \mathrm{ml}$ respectively. They also found "unopened bottles of the same lot were sterile" and "no infections could be attributed to contaminated soap in the ICU." They cite the literature as: "Several reports of intrinsic and extrinsic contamination of commonly used antiseptics and soaps such as chlorhexidine, hexachlorophene, and iodophors have appeared over the past decade." Apparently 2 of 23 bottles of OR Scrub did become contaminated under use conditions. Huntington concluded that OR Scrub needed a more effective preservative system.

In October 1983, Huntington began shipping OR Scrub with an improved preservative system; therefore, any OR Scrub now in use con- tains the new preservative system and is not the product which is the subject of this article in the September issue. ${ }^{1}$

OR Scrub is a surgical scrub and as such is used at full strength; it is not diluted prior to use. In fact, OR Scrub is an oil-water emulsion of antimicrobials, preservatives, surfactants, and emollients; it cannot be diluted with large amounts of water without breaking the emulsion into an oil layer and a water layer. Not only is the concentration of the antimicrobials and preservatives reduced when diluted, but the synergistic interaction of the oil-surfactant-antimicrobial system is destroyed also. In dilution the antimicrobial-preservative system may be in one layer and at least some of the microbial challenge in the other layer. Because of this separation, an accurate assessment of the preservative efficacy of the whole undiluted formulation cannot be extrapolated from tests on dilutions of OR Scrub. The USP Antimicrobial Preservative-Effectiveness Test protocol states "tests and standards apply only to the product in the original unopened container in which it was distributed by the producer." 3 The other products, Hibiclens and Wash, reported in the article are not oil-water emulsions and do form homogeneous solutions upon dilution.

The real concern is the ability of $\mathrm{OR}$ Scrub as bottled (full strength) to rid itself of an outside contamination while in use. Barry et al reported in the Addendum to this article that their January 1984 work shows that the OR Scrub in use today (with the improved preservative system) surprisingly can be diluted 1:512 and still rid itself of a 15,000,000 organism/ml Serratia marcescens challenge within 24 hours; and that OR Scrub diluted 1:4 can rid itself of the same $15,000,000$ organism $/ \mathrm{ml}$ challenge within 1 hour. ${ }^{2}$ Certainly a possible outside contamination challenge of 100 to $600 \mathrm{organisms} / \mathrm{ml}$ to full strength OR Scrub is no longer a concern.

The Barry et al ${ }^{1}$ modified preservative tests measure only a product's bactericidal properties; they do not measure a product's bacteriostatic or substantive properties which are very important attributes of a good surgical scrub. In vivo glove juice studies are the accepted protocols for measuring the efficacy of surgical scrubs. Glove juice studies show that OR Scrub is an efficacious surgical scrub.

Huntington is confident that OR Scrub is a good antimicrobial surgical scrub with pleasing cosmetic properties. Many hospitals have used OR Scrub successfully for 12 years.

\section{REFERENCES}

1. Barry MA, Craven DE, Goularte TA, et al: Serratia marcescens contamination of antiseptic soap containing triclosan. Implications for nosocomial infection. Infect Control 1984; 5:427-430

2. Barry MA, Goularte TA, Lichtenberg D, et al: OR Scrub: Should It Be Used in Operating Rooms and Intensive Care Units. Association for Practitioners in Infection Control, Annual Meeting, Board 52, abstracted. San Diego, CA, May 1-5, 1983.

3. Antimicrobial preservatives-effectiveness. United States Pharmacopeia 1980, pp XX, 51, 873-874.

F.M. Boyd, PhD Director of Research and Development Huntington Laboratories, Inc. Huntington, Indiana

\section{To the Editor:}

We have recently read the article by Barry et al concerning contamination of antiseptic soap containing triclosan. While we appreciate the authors' concern about potential nosocomial infection problems and also the importance of proper handwashing in hospitals, we have some concerns about their study and conclusions.

The subject of "proper handwashing" in health care institutions is one that entails considerable problems and complexities. As the authors point out in their article, handwashing in hospitals is often forgotten or performed inadequately. Also identified 